\title{
Scientific evidence in surgery for the treatment of temporomandibular joint internal derangement
}

\author{
Raúl González-García \\ Department of Oral and Maxillofacial-Head and Neck Surgery, University Hospital of Badajoz, Badajoz 06080, Spain. \\ Correspondence to: Dr. Raúl González-García, Calle Los Yébenes 35, 8C, Madrid 28047, Spain. E-mail: raulmaxilo@gmail.com
}

How to cite this article: González-García R. Scientific evidence in surgery for the treatment of temporomandibular joint internal derangement. Stomatological Dis Sci2019;3:5. http://dx.doi.org/10.20517/2573-0002.2018.26

Received: 15 Nov 2018 First Decision: 14 Mar 2019 Revised: 8 Apr 2019 Accepted: 15 Apr 2019 Published: 29 May 2019

Science Editors: Nikolaos G. Nikitakis, Dimitrios Dionysopoulos Copy Editor: Cai-Hong Wang Production Editor: Huan-Liang Wu

\begin{abstract}
Temporomandibular joint (TMJ) internal derangement (ID) is the most prevalent indication for surgery among TMJ pathology. Generally considered the literature lacks studies with the highest evidence -which is mainly supported by systematic reviews and randomized controlled trials- regarding the usefulness of surgery in the management of TMJ ID. The objective of the present manuscript is to report the actual knowledge in terms of surgical treatment of TMJ ID considering scientific evidence criteria. A non-systematic review in the literature in relation to the degree of the existing scientific evidence regarding the uselfuness of surgery for the treatment of ID of the TMJ is performed. A bibliography search on MEDLINE, EMBASE and the Cochrane library databases for studies published from March 2000 to March 2019 was conducted. The author only included studies published in the English language and those dealing with "surgical treatment of temporomandibular internal derangement"; The following technical bibliographic exclusion criteria were applied: (1) case reports; (2) technical report; (3) animal or in vitro studies; (4) review articles; (5) uncontrolled clinical studies; and (6) publications in which the same data were published by the same group of researchers. The abstracts of yielded results were reviewed and the full text of those with apparent relevance was obtained. Several results must be highlighted from the evaluation of the literature with the highest degree of evidence: (1) high-degree evidence is insufficient in relation to the effect of surgery; (2) surgery is useful in patients refractory to conservative treatment; (3) there are not differences among surgical techniques; (4) arthroscopy and arthrocentesis are effective in the treatment of chronic closed lock of the TMJ. Surgical treatment of the TMJ based on evidence is present in a moderate degree, as studies with the highest evidence are scarce. Specially for patients with TMJ ID, surgical treatment must be highly supported by the results of large series by experienced surgeons and recommendations from societies of experts in TMJ surgery.
\end{abstract}

Keywords: Evidence-based medicine, temporomandibular joint internal derangement, surgical treatment

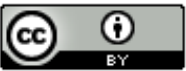

(C) The Author(s) 2019. Open Access This article is licensed under a Creative Commons Attribution 4.0 International License (https://creativecommons.org/licenses/by/4.0/), which permits unrestricted use, sharing, adaptation, distribution and reproduction in any medium or format, for any purpose, even commercially, as long as you give appropriate credit to the original author(s) and the source, provide a link to the Creative Commons license, and indicate if changes were made.

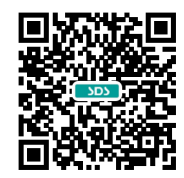




\section{INTRODUCTION}

Many temporomandibular joint (TMJ) pathologies have been reported to be subsidiary for surgical treatments, such as degenerative joint disease, rheumatoid or infectious arthritis, mandibular recidivant luxation, anchylosis, condylar hiper-/hypo-plasia, and tumors, being internal derangement (ID) the most prevalent and the focus of the present review [Figure 1]. According to the American Association of Oral and Maxillofacial Surgeons, surgery of the TMJ is indicated when: (1) conservative treatment has failed and pain or disfunction are moderate to severe; (2) quality of life is worsened with conservative treatment; and/ or (3) prognosis is worse if the disease is left to its natural evolution. Also, it is supported by the presence of disc displacement in the magnetic resonance imaging, although this condition is not imperative in terms of surgical indication. Meanwhile, surgery is clearly not indicated in: (1) asymptomatic or scarcely-symptomatic patients; and (2) in patients without pain and with satisfactory mandibular function.

The Evidence-Based Medicine (EBM) is defined as an approach to medical practice intended to optimize the decision-making by emphasizing the use of evidence from well-designed and well-conducted research. It may be necessary to make a precise question, search proofs in the literature, make a critical evaluation of the evidence, and wether it is found adequate, applicate this new knowledge to the practice [Figure 2]. Generally speaking, literature regarding treatment of TMJ disease lacks of studies with a high degree of evidence, which is mainly supported by systematic reviews (SRs) and randomized controlled trials (RCTs).

In the present paper the author makes a non-systematic review of the literature in relation to the degree of the existing scientific evidence regarding the uselfuness of surgery for the treatment of ID of the TMJ, in order to provide an overall view of the state of the art regarding this topic.

\section{BIBLIOGRAPHY SEARCH}

A bibliography search on MEDLINE, EMBASE and the Cochrane library databases for studies published from March 2000 to March 2019 was conducted, with the searching terms: ("temporomandibular joint disease" [MeSH Terms]) OR ("temporomandibular joint dysfunction" [MeSH Terms]) OR ("temporomandibular joint síndrome" [MeSH Terms] AND "surgery" [All Fields]) OR ("surgical treatment" [All Fields]) OR ("arthroscopy" [All Fields]) OR ("arthroscopic surgery” [All Fields]). References were explored to identify other articles.

Only those studies published in the English language and those dealing with "surgical treatment of the temporomandibular joint internal derangement" were selected, excluding those referring to other temporomandibular joint diseases not specifically classified as TMJ ID. Then, a manual screening of articles' abstracts was performed in order to explore the role of surgery in the management of internal derangement or dysfunction of the TMJ from the ultimate complete 19 years. The following technical bibliographic exclusion criteria were applied: (1) case reports; (2) technical reports; (3) animal or in vitro studies; (4) review articles; (5) uncontrolled clinical studies; and (6) non-surgical methods were used for managing TMJ ID, including arthrocentesis. The abstracts of yielded results were reviewed and the full text of those with apparent relevance was obtained. The references of identified articles were crosschecked for unidentified articles. The author carefully assessed the eligibility of all studies retrieved from the databases. A total amount of 12 original papers were finally selected according to the provided inclusion and exclusion criteria $^{[1-12]}$.

\section{EVIDENCE IN SURGICAL TREATMENT OF TMJ ID}

\section{Main schemes in EBM clasiffication}

Regarding classification, the US Agency for Healthcare Research and Quality (AHRQ) classified the levels of scientific evidence in: (1) 1a-SRs of RCTs; (2) 1b-at least 1 RCT; (3) 2a-at least 1 well-designed controlled 


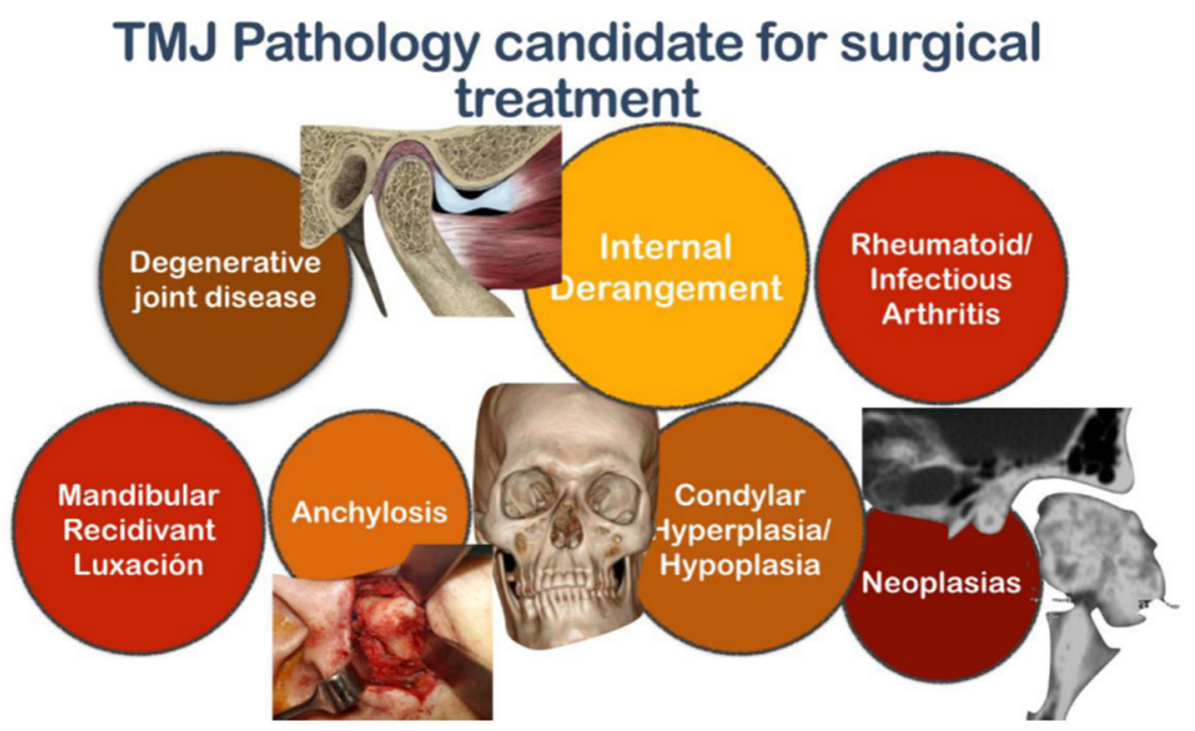

Figure 1. Temporomandibular joint pathologies candidate for surgical treatment

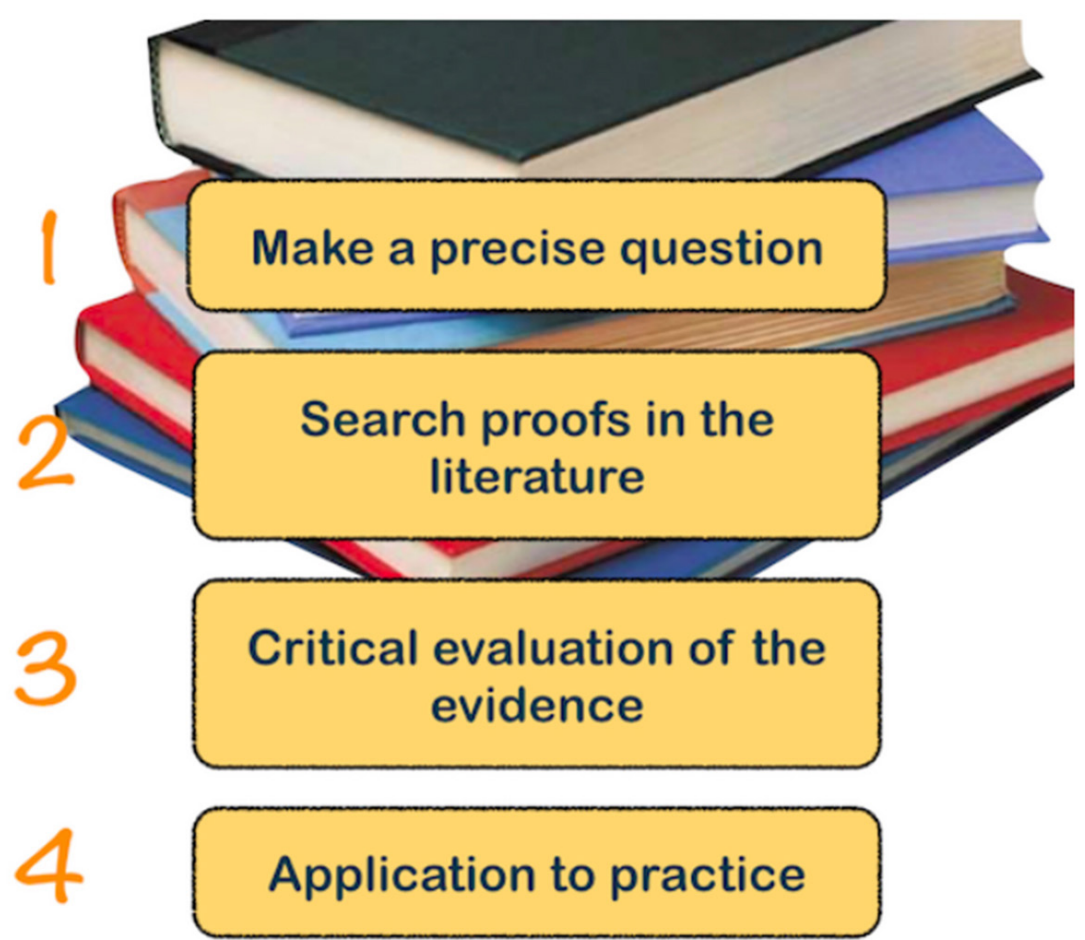

Figure 2. Global steps in Evidence-Based Medicine

study without randomization; (4) 2b-at least 1 other type of well-designed quasi-experimental study; (5) 3-well-designed non-experimental descriptive studies such as comparative, correlation or cases-control; and (6) 4-expert committee reports or opinions [Figure 3].

Also, the Oxford Centre for Evidence-Based Medicine (OCEBM) decided to report on its classification system. In the highest level of evidence the SRs of RCTs are found, followed by individual RCTs; in a second degree of evidence, SRs of cohort studies followed by individual cohort studies are placed; in a third degree 


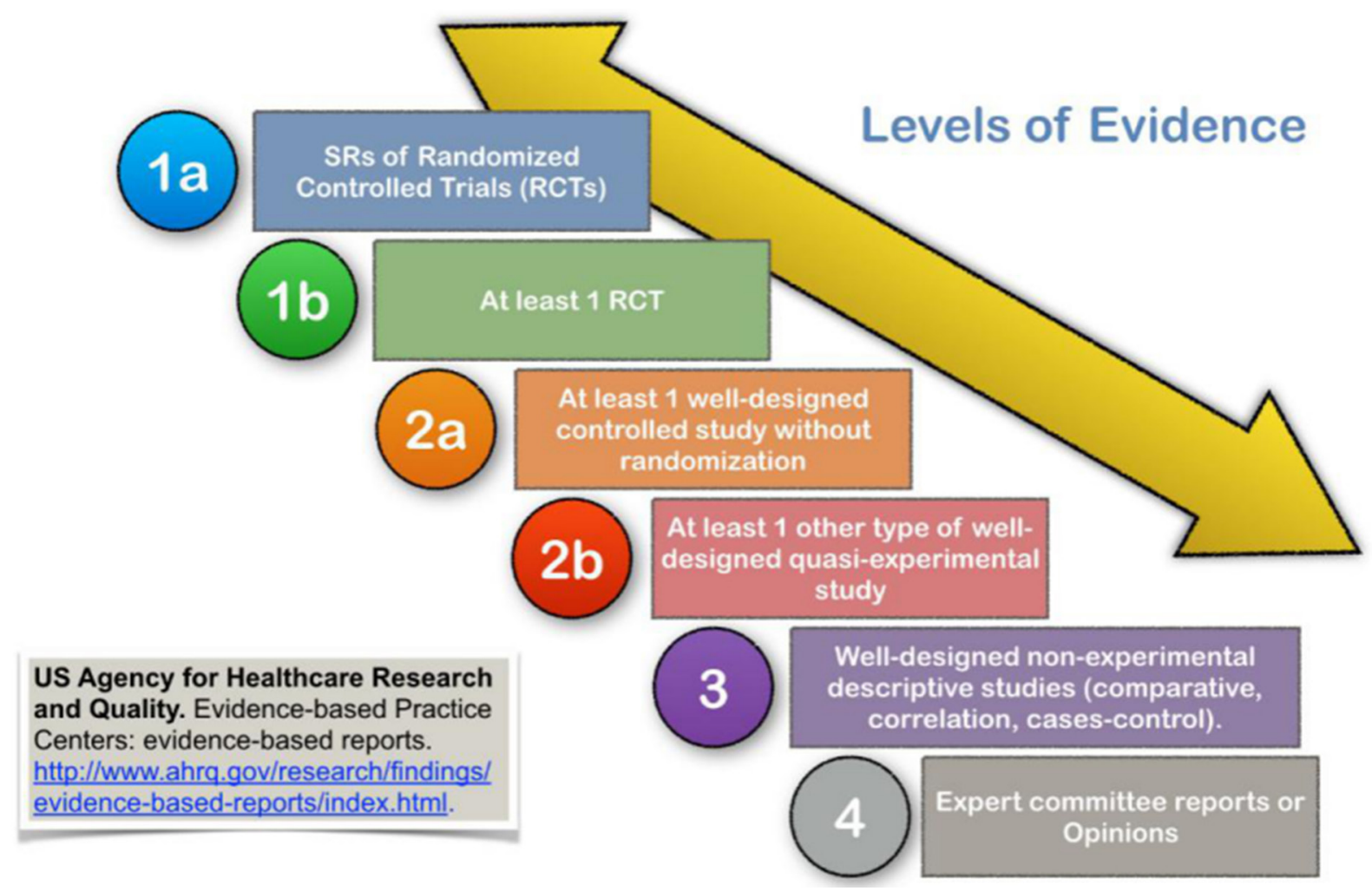

Figure 3. Levels of evidence by the US Agency for Healthcare Research and Quality

of evidence SRs of case-controlled studies followed by individual case-control studies are found; last, case series and expert opinions occupy the lowest steps in terms of evidence [Figure 4].

Other widely used systems for hierarchying the evidence are those from the National Health and Medical Research Council, the National Institute for Health and Clinical Excellence, the Canadian Task on Force on the Periodic Health Examination, the United States Preventive Services Task Force, and the Scotish Intercollegiate Guidelines Network, among others.

\section{SRs}

Summarizing the philosophy undergoing the usefulness of surgical treatment of TMJ ID, a letter by Hall ${ }^{[1]}$ approached the results by some meta-analyses and RCTs about pain relief in TMJ surgical procedures, establishing that prompt relief of pain was one of the chief reasons for most patients looking for surgical treatment. As reminded in author's comment, a placebo effect may account for some of the improvement after operation, but it should be expected no more than 30\%-35\% of the pain relief. These findings may justify that surgery may be undoubtedly responsable for such improvement in terms of pain. This autor also concluded that although evidence was imperfect, major joint operations were effective in the management of the painful TMJ with ID.

When considering SRs or evidence 1a in both the AHRQ and the OCEBM classifications, some relevant papers were found, such as the one by Reston and Turkelson ${ }^{[2]}$ in 2003, who evaluated the effectiveness of different surgical modalities in the treatment of TMJ ID: arthrocentesis, arthroscopy, discectomy and discopexy. They concluded that: (1) surgical treatments were beneficial for patients who were refractory to conservative treatments; (2) there were no differences between the analyzed surgical options; and (3) there was evidence about the effectiveness of arthrocentesis and arthroscopy for the treatment of patients with disc displacement without reduction (DDwoR). 


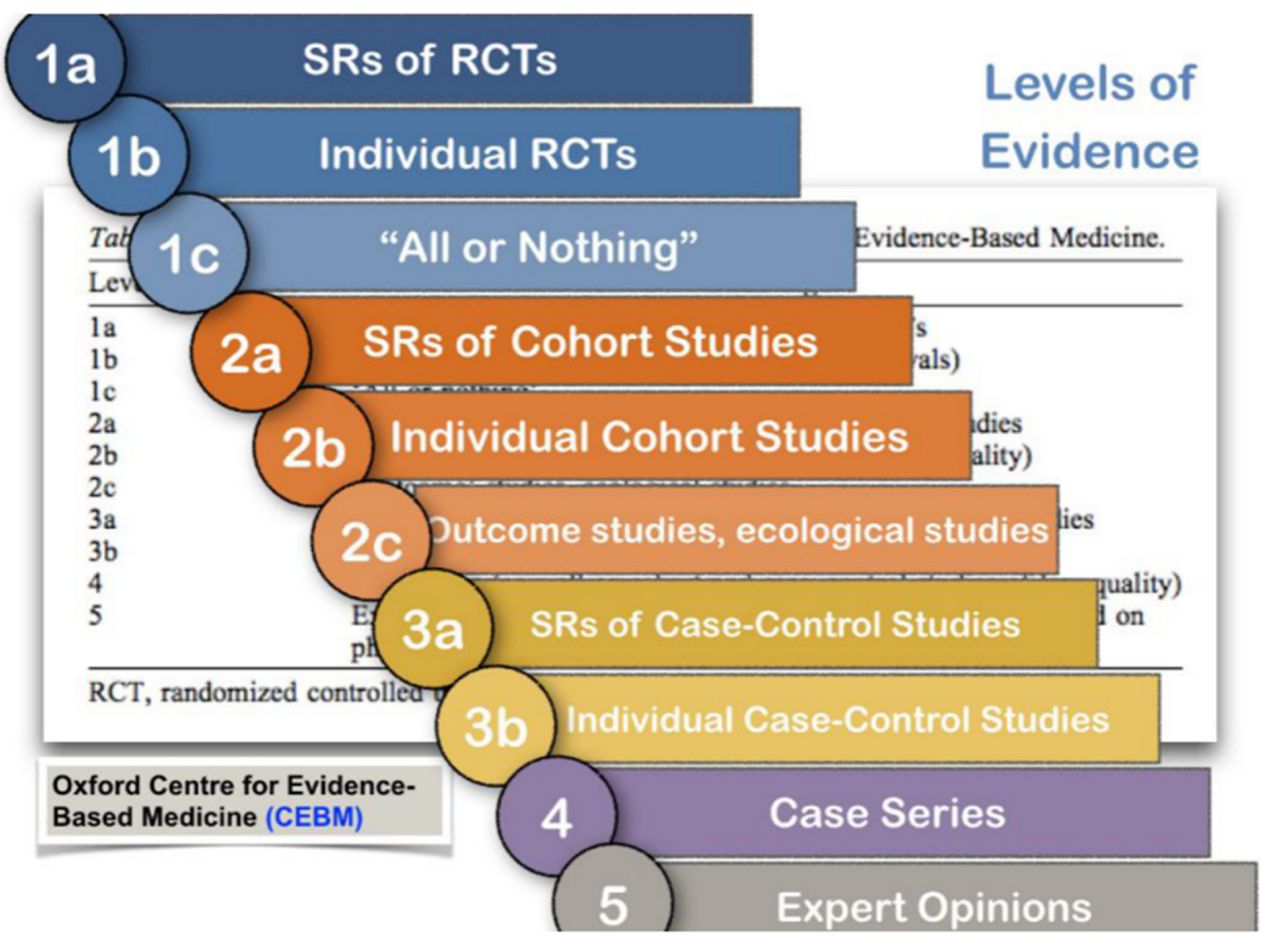

Figure 4. Levels of evidence by the Oxford Centre for Evidence-Based Medicine

In 2010, List and Axelsson ${ }^{[3]}$ tried to evaluate the evidence and quality of SRs published in relation to the treatment of TMJ disorders. A total amount of 30 studies were included, 23 of them qualitative and 7 quantitative. Among them, only 3 studies approached the surgical treatment of TMJ ID, which is a clear indication of the paucity of SRs dealing with surgical treatment of TMJ ID. In the group of patients with DDwoR, the comparison between arthrocentesis, arthroscopy and discectomy did not show any statistical difference. Also, in the group of patients with disc displacement with reduction (DDwR) there were not differences between arthrocentesis, arthroscopy and conservative treatment by physiotherapy. These authors concluded that evidence was not enough in relation to the effect of surgery, while the main limitation of the analyzed SRs was that the variability of the primary studies did not allow to establish firm conclusions.

Only one year later, Rigon et al. ${ }^{[4]}$ performed a SR dealing with the effectiveness of arthroscopy vs. open surgery, arthrocentesis and conservative treatment in TMJ ID, in relation to pain, function and clinical signs. They found 7 RCTs and 349 patients meeting the inclusion criteria. They concluded that in patients with closed lock, open surgery reduced pain more than arthroscopy, from the 12th month post-operatively. Also, they reported that, in patients with DDwR or DDwoR, arthroscopy obtained better results in terms of mouth opening than arthrocentesis, from the 12th month post-operatively.

Besides, De Souza et al. ${ }^{[5]}$ in a SR of the published literature, reviewed the evidence concerning several treatments for TMJ osteoarthritis. They only found 3 RCTs comparing different treatments. Their results were that: (1) there were no differences between the injection of sodium hyaluronate and the injection of corticoids; (2) there were no differences between diclofenac sodium an occlusal splint therapy; and (3) there were no differences between glucosamine sulfate and ibuprofen. The concluded that there was a noticeable lack of RCTs dealing with surgical treatments and that the lack of data from RCTs made decision-making about the management of TMJ osteoarthritis strongly dependent on consumers'preferences and clinical expertise. 
Fricton et al.$^{[6]}$ reviewed the evidence concerning several treatments for TMJ disorders. They performed a quality assessment of 210 RCTs checking the internal and external validity using the Consolidated Standards of Reporting Trials criteria. They concluded that the overall quality of the reviewed studies was modest, with only $58 \%$ of the quality criteria met, while only $10 \%$ of the RCTs met the four most important criteria. In contrast to these discouraging data, they also assessed that there had been a trend towards improvement in studies quality over time.

To assess wether arthroscopy or arthrocentesis was most effective, Al-Moraissi ${ }^{[7]}$ performed a SR and metaanalysis on the management of TMJ ID. Two RCTs, 2 non-randomized clinical controlled trials, and 2 cases series were included. Based on this work, the author concluded that arthroscopy was observed to be superior than arthrocentesis in increasing joint movement and decreasing pain, while both arthroscopy and arthrocentesis had comparable post-operative complication rates. The main limitation of this study was that meta-analysis was incomplete due to the paucity of good quality studies in the high-impact, peer-reviewed literature.

\section{RCTs}

When considering individual RCTs or evidence $1 \mathrm{~b}$ in both the AHRQ and the OCEBM classifications, a few relevant papers were found. Holmlund et al. ${ }^{[8]}$ compared the effectiveness of open surgery by discectomy vs. arthroscopic lysis and lavage (ALL) in 22 patients with DDwoR. They concluded that both open surgery (by discectomy) and ALL were equally effective in the treatment of DDwoR and that, as far as arthroscopy was a minimally-invasive procedure, it should be considered the first choice among surgical treatments.

With the same approach in 2007, Politi et al. ${ }^{[9]}$ compared the effectiveness of open surgery, in this series by high condilectomy and disc reposition, vs. ALL, in 20 patients with DDwoR under the design of a RCT. They concluded that both open surgery (by high condilectomy plus disc reposition) and ALL were equally effective in the treatment of DDwoR. As the previous authors, they recommended arthroscopy as the first choice among surgical options.

When specifically dealing with arthroscopic intraarticular injection of substances, Fernández Sanromán et al. ${ }^{[10]}$ in a randomized prospective clinical study in 92 patients with Wilkes stage IV internal derangement, showed significant reduction of pain and significant increase in mouth opening for both groups of patients treated either with plasma rich in growth factors (PRGF) or with saline solution, with no differences among treatment modalities. They concluded that the injection of PRGF did not add any significant improvement to clinical outcomes at 2 years post-op in those patients with advanced disease. However, in a posterior study by the same group, Fernández-Ferro et al ${ }^{[11]}$ in another randomised prospective clinical study with a total of 100 patients with ID with osteoarthritis treated arthroscopically either with intra-articular injection of PRGF or hyaluronic acid (HA), concluded that the injection of PRGF following arthroscopy was more effective than the injection of HA regarding pain reduction, although no statistical differences wer observed in relation to the increase of mouth opening, being both methods effective in the management of advanced ID.

In contrast to most reported RCTs, Schiffman et al. ${ }^{[12]}$ compared the effectiveness among 4 treatment options in patients with chronic closed lock (CCL) or DDwoR: drugs, physiotherapy, arthroscopy and open surgery. They concluded that in patients with CCL, short term improvement ( 3 months) in terms of pain and function was equal for the 4 treatment options, while no additional improvement from surgery over conservative treatment was observed; for these authors primary treatment of DDwoR should consist on drug therapy or physiotherapy. Now this asseveration is difficult to maintain for patients with CCL of the TMJ, as it has been clinically observed that function in terms of mouth opening does not substantially improve 
by only conservative treatments in this group of patients. Moreover, the inclusion of this RCT as part of posteriorly published SRs may have had a clear deletereous effect in trying to probe the usefulness of pure surgical options. The major criticism to the conclusions of the previously mentioned study is that it grossly understimate the observed benefits of surgical treatments for TMJ ID, which have been extensively reported in many clinical series, specially for those patients who are refractory to conservative options.

At this point, it is convenient to remember that when dealing with surgical fields, particularly regarding TMJ surgery, it is very difficult to elaborate recommendations from studies with the highest degree of evidence. To illustrate this idea, Smith and Pell ${ }^{[13]}$ performed a SR of RCTs dealing with the effectiveness on the use of the parachute to prevent damage from gravitational falling. Not surprisingly, they found that its usefulness had not been yet demonstrated by the EBM. This is the reason why in normal clinical practice evidence present in $2 \mathrm{~b}$ and 3 well-designed studies are stimated enough to guide the clinician in relation to the surgical treatment of TMJ ID.

\section{OVERALL REPORT}

While the highest levels of evidence are strongly supported in many medical fields, Gonçalves et al. ${ }^{[14]}$ and Harris et al. ${ }^{[15]}$ have stated that scientific evidence $2 \mathrm{~b}$ and 3 should be considered as evidence enough to guide clinical protocols in many surgical areas, including Oral and Maxillofacial Surgery. Thus, well-designed quasi-experimental (defined by a broad range of non-randomized intervention studies, usually made when it is not logistically feasible or ethical to conduct a RCT) and well-designed non-experimental descriptive studies are of undoubtful usefulness in our field.

This general idea was also supported by Melo et al. ${ }^{[16]}$ who stated that when studies with a high level of evidence on a given subject are not available, observations from case reports and case series can provide clinical information for consideration by the surgical community until cohort studies or RCTs can be conducted.

Based on the present review, the studies with highest evidence conclude that: (1) high-degree evidence is insufficient in relation to the effect of surgery; (2) surgery is useful in patients refractory to conservative treatment; (3) there are not differences among surgical techniques; (4) arthroscopy and arthrocentesis are effective in the treatment of chronic closed lock. Although both open surgery and arthroscopy are effective in treating patients with TMJ internal derangement, arthroscopy is preferred as the first option due to its non-invasive nature.

For a deepest knowledge of this field, the TMJ surgeons' community should encourage researchers to: (1) design new randomized controlled trials (RCTs); (2) include patients with accurate diagnoses and categorized by Wilkes' stages; (3) eliminate bias in relation to Cochrane recommendations for elaborating RCTs; (4) design multi-center studies based on accurate clinical practice protocols; (5) elaborate clinical guides by National and International Expert Committees.

\section{CONCLUSION}

In summary, surgical treatment of the TMJ based on evidence is present in a moderate degree, as studies with the highest evidence are scarce. Specially for patients with TMJ internal derangement, surgical treatment is highly supported by the results of large series by experienced surgeons, and the recommendations from societies of experts in TMJ surgery such as the European Society of TMJ Surgeons (ESTMJS) and the American Society of TMJ Surgeons. 


\section{DECLARATIONS}

\section{Authors' contributions}

González-García R contributed solely to this sthdy.

\section{Availability of data and materials}

Not applicable.

\section{Financial support and sponsorship}

None.

\section{Conflicts of interest}

The author declared that there are no conflicts of interest.

\section{Ethical approval and consent to participate}

Not applicable.

\section{Consent for publication}

Not applicable.

\section{Copyright}

(C) The Author(s) 2019.

\section{REFERENCES}

1. Hall HD. Temporomandibular joint surgery meta-analysis; pain relief as an outcome measure. J Oral Maxillofac Surg 2003;61:851.

2. Reston J, Turkelson CM. Meta-analysis of surgical treatment for temporomandibular articular disorders. J Oral Maxillofac Surg 2003;61:310.

3. List T, Axelsson S. Management of TMD: evidence from systematic reviews and meta-analisis. J Oral Rehabil 2010;37:430-51.

4. Rigon M, Pereira LM, Bortoluzzi MC, Loguercio AD, Ramos AL, et al. Arthroscopy for temporomandibular disorders. Cochrane Database Syst Rev 2011;11:CD006385.

5. De Souza RF, Lovato da Silva CH, Nasser M, Fedorowicz Z, Al-Muharraqi MA. Interventions for the management of temporomandibular joint osteoarthritis. Cochrane Database Syst Rev 2012;18:CD007261.

6. Fricton JR, Ouyang W, Nixdorf DR, Schiffman EL, Velly AM, et al. Critical appraisal of methods used in randomized controlled trials of treatments for temporomandibular disorders. J Orofac Pain 2010;24:139-51.

7. Al-Moraissi EA. Arthroscopy versus arthrocentesis in the management of internal derangement of the temporomandibuar joint: a systematic review and meta-analysis. Int J Oral Maxillofac Surg 2015;44:104-12.

8. Holmlund AB, Axelsson S, Gynther GW. Comparisson of discectomy and arthroscopic lysis and lavage for the treatment of chronic closed lock of the temporomandibular joint: a randomized outcome study. J Oral Maxillofac Surg 2001;59:972-7.

9. Politi M, Sembronio S, Robiony M, Costa F, Toro C, et al. High condylectomy and disc repositioning compared to arthroscopic lysis, lavage, and capsular stretch for the treatment of chronic closed lock of the temporomandibular joint. Oral Surg Oral Med Oral Pathol Oral Radiol Endod 2007;103:27-33.

10. Fernández Sanromán J, Fernández Ferro M, Costas López A, Arenaz Bua J, López A. Does injection of plasma rich in growth factors after temporomandibular joint arthroscopy improve outcomes in patients with Wilkes stage IV internal derangement? A rendomized prospective clinical study. Int J Oral Maxillofac Surg 2016;45:828-35.

11. Fernández-Ferro M, Fernández-Sanromán J, Blanco-Carrión A, Costas-López A, López-Betancourt A, et al. Comparison of intra-articular injection of plasma rich in growth factors versus hyaluronic acid following arthroscopy in the treatment of temporomandibulardysfunction: a randomised prospective study. J Craniomaxillofac Surg 2017;45:449-54.

12. Schiffman EL. Randomized effectiveness study of four terapeutic strategies for TMJ closed lock. J Dent Res 2007;86:58-63.

13. Smith GCS, Pell JP. Parachute use to prevent death and major trauma related to gravitational challenge: a systematic review of randomized clinical trials. BMJ 2003;327:1459-61.

14. Gonçalves JR, Cassano DS, Rezende L, Wolford LM. Disc repositioning: does it really work? Oral Maxillofac Surg Clin North Am 2015;27:85-107.

15. Harris AD, McGregor JC, Perencevich EN, Furuno JP, Zhu J, et al. The use and interpretation of quasi-experimental studies in medical informatics. J Am Med Inform Assoc 2006;13:16-23.

16. Melo AR, Pereira Júnior ED, Santos LAM, Vasconcelos BCDE. Recurrent dislocation: scientific evidence and management following a systematic review. Int J Oral Maxillofac Surg 2017;46:851-6. 